\title{
Acute Hydrocephalus Revealing Unusual Cerebellar Mass: Dysplastic Cerebellar Gangliocytoma or Lhermitte-Duclos Disease (LDD)
}

\section{Karekezi*, M. Boutarbouch, O. Coulibaly, S. Derraz, A. El Ouahabi, A. El Khamlichi}

Department of Neurosurgery, Mohamed Vth University, School of Medicine, CHU IBN SINA, Hôpital des Spécialités ONO, Rabat, Morocco.

Email: *clairekarekezi@gmail.com

Received April $8^{\text {th }}, 2013$; revised May $12^{\text {th }}, 2013$; accepted June $14^{\text {th }}, 2013$

Copyright (C) 2013 C. Karekezi, et al. This is an open access article distributed under the Creative Commons Attribution License, which permits unrestricted use, distribution, and reproduction in any medium, provided the original work is properly cited.

\begin{abstract}
Lhermitte-Duclos disease (LDD) or dysplastic gangliocytoma of the cerebellum is a rare benign lesion of uncertain pathogenesis characterized by overgrowth of cerebellar ganglion cells which replace granular cells and Purkinje cells; this results in gross thickening of the cerebellar folia. It is revealed by symptoms of raised intracranial pressure, cerebellar impairment and obstructive hydrocephalus. We reported the case of a 41-year-old male who presented with symptoms of acute raised intracranial pressure. Brain computed tomography (CT) scan revealed hydrocephalus due to compression of the 4th ventricle by a large non-enhancing left cerebellar mass. Magnetic resonance imaging (MRI) showed a space-occupying lesion within the left cerebellar hemisphere with unusual striation. Radical surgery was retained though the margins with normal cerebellar tissue were not distinct. Clinical complications after gross total or partial removal of Lhermitte-Duclos lesions have been rarely reported in the literature; herein we stress the importance of extreme caution in removing these lesions in cerebellar areas that have no distinct margins between the lesion and normal tissue.
\end{abstract}

Keywords: Lhermitte-Duclos Disease; Dysplastic Gangliocytoma; MRI; Cerebellar Tiger Stripes

\section{Introduction}

Lhermitte-Duclos disease (LDD), or dysplastic gangliocytoma, is a rare benign condition of uncertain nature involving the cerebellum, first described by Lhermitte and Duclos in 1920 [1]. LDD is characterized by overgrowth of cerebellar ganglion cells which replace granular cells and Purkinje cells, resulting in gross thickening of the cerebellar folia $[1,2]$.

In the past, this rare entity was associated with very poor prognoses; approximately one third of patients died from raised intracranial pressure due to mass effect from lesion and acute hydrocephalus [3]. Subsequently, Lhermitte-Duclos disease has become a surgically treatable and even curable condition. One of the major challenges for the surgeon during surgical exploration of LhermitteDuclos cerebellar tumors is the lack of clear margins with the normal cerebellar tissue which are often not well defined [3-5].

\footnotetext{
"Corresponding author.
}

The goal of this article is to report the advanced magnetic resonance imaging (MRI) findings in a case of LDD associated and point out the importance of extreme caution in removing these lesions with no clear limits to the normal cerebellar tissue.

\section{Case Report}

We reported the case of a 41-year-old male who presented with acute onset of severe headache, followed by vertigo, fall and short loss of consciousness admitted to the emergency department, disoriented with bilateral Papilledema at fundoscopy.

Head CT scan revealed hydrocephalus due to compression of the 4th ventricle by a large left cerebellar non-enhancing mass (Figure 1).

MRI sequences showed a large oedematous left cerebellar hemisphere with a mass lesion of abnormal signal intensity in the region of the vermis. On T1-weighted images, the mass was predominantly hypointense and had typical prominent hypo- and unusual striations on 
T2-weighted images the mass was predominantly hyperintense and had isointense striations. On MR spectroscopy, N-acetyl aspartate (NAA), cholin (Cho), creatinine $(\mathrm{Cr})$ were elevated and $\mathrm{NAA} / \mathrm{Cr}$, $\mathrm{Cho} / \mathrm{Cr}$ ratios were found slightly elevated (Figures 2(a)-(d)).

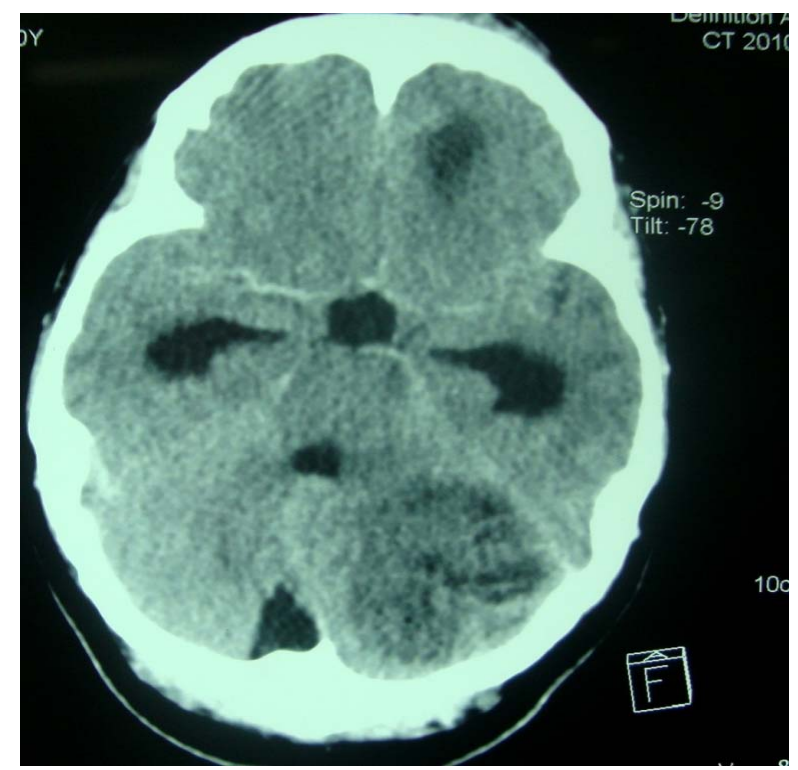

Figure 1. Contrast-enhanced axial computed tomography (CT) scan showing hypodense, non-enhancing left cerebellar lesion compressing the fourth ventricle causing hydrocephalus.

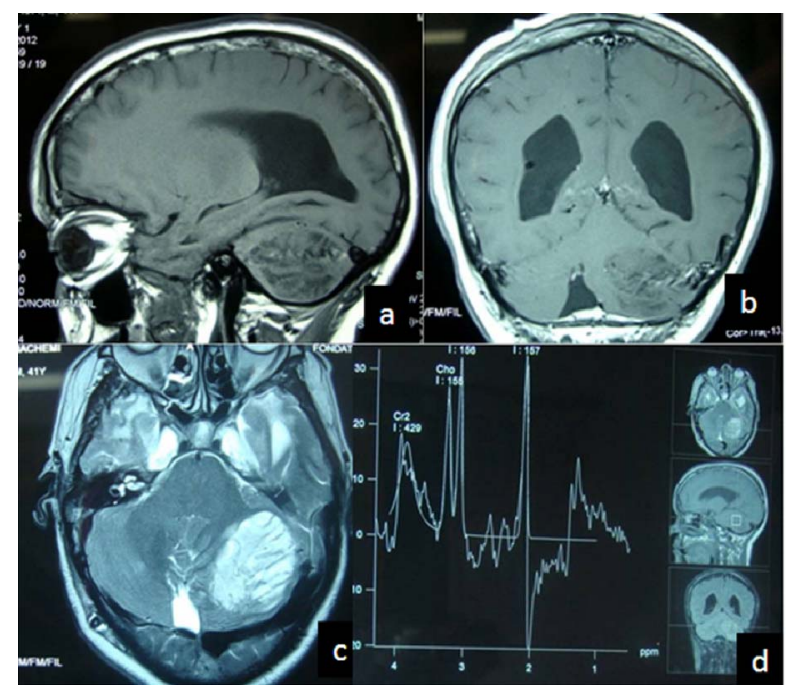

Figure 2. (a) T1 Sagittal MRI showing hypointense right cerebellar lesion with linear striations; (b) T1 coronal MRI showing a non-enhanced hypointense-to-isointense right cerebellar lesion with linear striations; (c) axial MRI showing a hyperintense lesion on T2-weighted MRI with areas of linear hypointensity streaks running throughout lesion (d) MR spectroscopy: N-acetyl aspartate (NAA), cholin (Cho), creatinine $(\mathrm{Cr})$ elevated and $\mathrm{NAA} / \mathrm{Cr}, \mathrm{Cho} / \mathrm{Cr}$ ratios were found slightly elevated.
The patient underwent an emergent ventricular peritoneal shunting for hydrocephalus with prompt improvement of signs of raised intracranial pressure. At day 8 post shunting, patient underwent suboccipital craniectomy and C-1 laminectomy with resection of the left cerebellar lesion. During the operation, the poorly demarcated lesion was hardly excised from the apparently normal surrounding cerebellar tissue; the margins with normal cerebellar tissue were not clearly distinct resulting in subtotal removal (Figure 3).

Histological examination showed diffuse replacement of tightly packed, small neurons of granule cell layer-

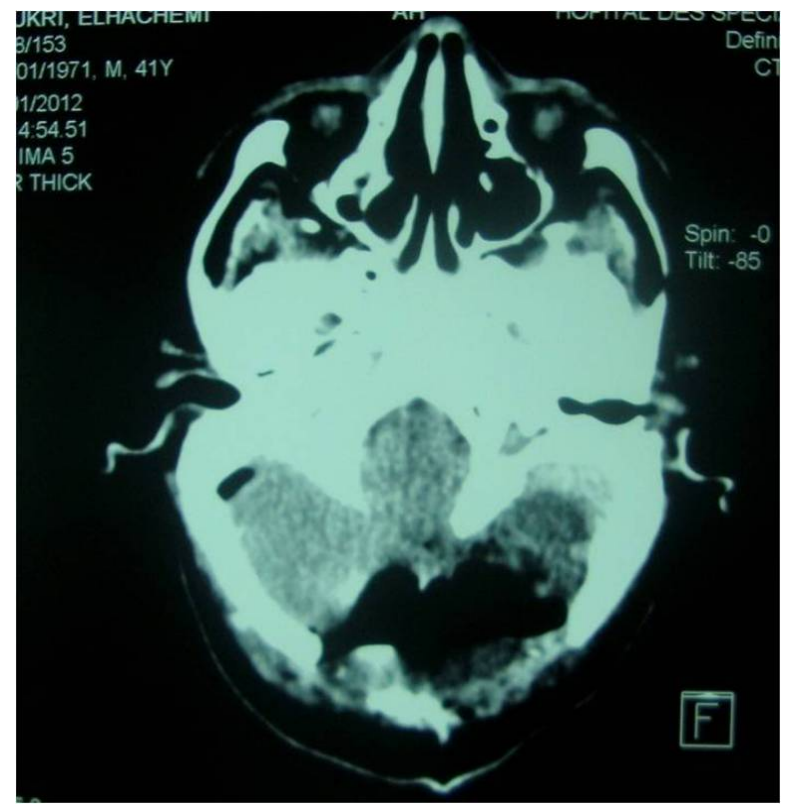

Figure 3. Axial CT scan showing partial resection of lesion with no clear limits with the normal tissue.

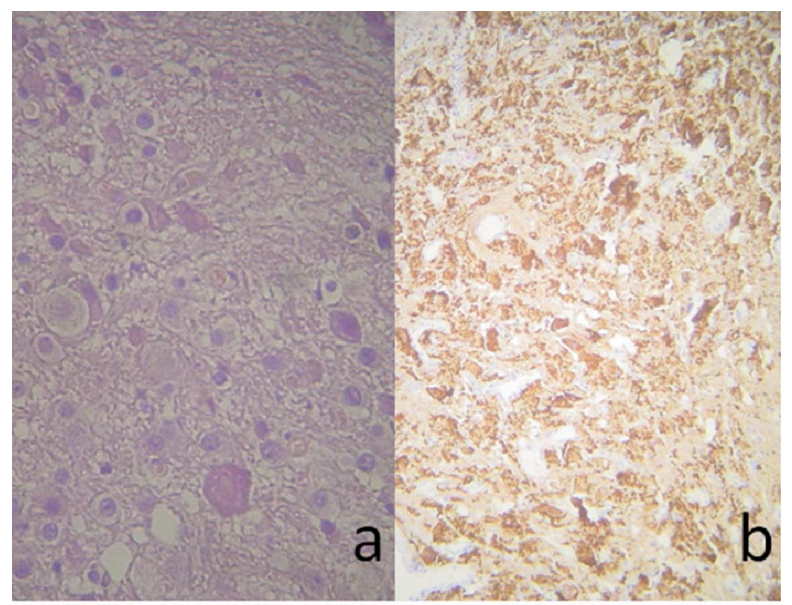

Figure 4. (a) Enlarged and distorted cerebellar folia withMolecular and internal granular layers Enlarged with dysplastic ganglion cells; (b) Dysplastic ganglion cells positive for synaptophysin. 
by large cells, with ganglion cell-like features, abnormally myelinated axons extended from granular layer to the overlaying molecular layer, which appeared expanded. Synaptophysin immunoreactivity was present in the large neuronal cells; Immunohistochemistry for endothelial markers revealed numerous venular spaces in leptomeninges, granular and molecular layers; confirming Lhermitte-Duclos Disease (Figures 4(a) and (b)).

Day two postoperative was marked by rapidly increased intracranial pressure with loss of consciousness. Brain CT scan revealed marked brain swelling in the posterior fossa with brainstem compression and herni ation without hydrocephalus. The patient remained deeply unconscious with a marked electrolyte imbalance. Death occurred before any emergent decompression.

\section{Discussion}

First described by Lhermitte and Duclos in 1920, rare benign lesion of uncertain pathogenesis; approximately 220 patients with LDD have been reported in the literature in 2006 [1]. LDD is characterized by overgrowth of cerebellar ganglion cells which replace granular cells and Purkinje cells resulting in gross thickening of the cerebellar folia. There is still considerable controversy over the cause of this disease: It is now considered to have a hamartomatous rather than a neoplastic origin though classified as a WHO grade I tumor $[2,3]$.

Lhermitte-Duclos has clinical evidence and close association with a multiple hamartoma-neoplasia complex known as Cowden syndrome [3]. Cowden syndrome has autosomal dominant inheritance and is characterized by a variety of mucocutaneous lesions, macrocephaly, and increased frequency of hamartomas and neoplasia in the breast, thyroid, colon, genitourinary organs, and central nervous system $[2,3]$. It is considered that patients with Cowden syndrome have germline mutations in the PTEN gene at locus 10q23.2, which has been identified as the major susceptibility gene for Cowden syndrome [2-4,7]. Hence, patients with Cowden syndrome should undergo an MR examination of the brain to rule out associated dysplastic cerebellar gangliocytoma and vice versa [4].

Dysplastic cerebellar gangliocytoma is seen most frequently in young adults (average age, 34 years). Less frequently, it occurs in pediatric patients [6-8]. There is no sex predilection. Clinically, patients may be asymptomatic, or they may present with symptoms and signs of increased intracranial pressure. Headache, vertigo, cerebellar symptoms and sudden neurologic deterioration as a result of occlusive hydrocephalus are frequent findings. The anamnesis finds long-standing symptoms that have been present for years that may point to the slowly progressive nature of this disease [9].
Imaging plays an important role in the diagnostic process, the dysplastic gangliocytoma is hypo-attenuated on unenhanced computed tomographic (CT) images $[10,11]$. MR imaging is the modality of choice, as it is for any posterior fossa abnormality, MR imaging reveals a cerebellar mass with a typical striated or tiger-striped folial pattern that consists of alternating bands on both T1and T2-weighted images, MR spectroscopy reveals reduced N-acetylaspartate-choline and N-acetylaspartatecreatine ratios compared with those of normal cerebellar tissue, lactate peak may also be present [11,14]. MR imaging is also invaluable for preoperative planning and as an aid to determine the extent of resection. In asymptomatic patients, MR imaging helps in the assessment of the lesion extent, mass effect, presence of hydrocephalus, and need to perform surgery. Regular follow-up is required $[13,14]$.

Lhermitte-Duclos disease clinical presentation follow the insidious expansion in the posterior cerebral fossa, surgery appears to be the only option relieve symptoms by especially in patients with raised intracranial pressure to avoid brain herniation and death. As the lesion is categorized as a WHO grade I tumor, a conservative "wait and see" strategy can be chosen especially in cases without elevated intracranial pressure. Clinical and radiologic follow-ups with MR imaging should be preferred in such cases $[15,16]$.

Drainage of the CSF by a ventricular shunt or by third endoscopic ventriculo-cisternostomy (ETV) might be preferred initially for the obstructive hydrocephalus and followed by tumor resection. However, complete resection may be difficult because of poorly defined margins. The lesion usually blends into normal cerebellar parenchyma and frequently, during surgical exploration, no tumour mass can be found, thus the absence of tumour limits in the depth of the cerebellar hemisphere constitutes the major technical problem during surgery.

This macroscopic appearance is confirmed by histopathological findings demonstrating a transitional area between normal and pathological cerebellar tissue. Histological examination reveals change in the normal cerebellar cortical cell layers with dysplastic hypertrophied ganglion cells leading to expansion of the granule layer and increased myelination in the molecular layer causing it to widen.

Because this transition from normal to pathological cerebellar tissue is gradual, accompanied by histologically verified disappearance of Purkinje cells and central white matter; the lack of a sharp border between the tumour and cerebellar tissue limits the accuracy of surgical excision. Lhermitte-Duclos tumours are not always completely removable lesions, for which normal recovery seems to be possible. 
Most patients have an uneventful postoperative period; some cases with post-operative posterior fossa brain swelling have been reported due to insufficiency tumor removal by lack of clear margins between normal tissue and the lesion; Yang Ms et al. [16] have reported a similar case with post-operative brain swelling aggravating the hydrocephalus resulting in an additional shunting; AfsharOromieh et al. reported a case of post-operative cerebellar mutism following a removal of LDD lesion, with difficulty to distinguish between tumor and healthy cerebellar tissue, resulting in extensive resection and neuronlogical deficits [17].

In accordance with previous observations, our case of Lhermitte-Duclos disease was macroscopically characterized by an indistinct border between the normal and tumour tissue. Nevertheless, surgical resection of the cerebellar mass is undoubtedly the correct course of treatment and clinical problems after gross macroscopic or total removal of the disease have rarely been reported in the literature. Surgical technics to distinguish the precise margins of the abnormal tissue of the tumour from the normal cerebellar tissue have to be discussed for the removal of these kinds of lesions.

\section{Conclusion}

In conclusion, LDD is a rare cause of posterior fossa masses characterized by symptoms of raised intracranial pressure and atypical, "tiger striped" pattern on conventional MRI. Complete surgical resection of the cerebellar mass remains undoubtedly the correct course of treatment but most of the time unachieved due to lack of distinct borders between the lesion and the normal cerebellar tissue. Clinical complications after gross macroscopic or total removal of the disease have rarely been largely discussed in the literature and are still a major challenge for the surgeon. Extreme caution should be taken in removing these lesions in cerebellar areas that have no distinct borders between the lesion and normal tissue. Incomplete removal of the mass may result in cerebellar swelling and clinical deterioration. Coexistence with Cowden syndrome should prompt thorough clinical examination and necessary investigations to detect or exclude concomitant malignancies.

\section{REFERENCES}

[1] S. Derrey, F. Proust, B. Debono, O. Langlois, A. Layet and V. Layet, "Association between Cowden Syndrome and Lhermitte-Duclos Disease: Report of Two Cases and Review of the Literature," Neurochirurgie, Vol. 52, No. 5, 2006, pp. 407-414. doi:10.1016/S0090-3019(03)00576-7

[2] S. Robinson and A. R. Cohen, "Cowden Disease and Lhermitte-Duclos Disease: Characterization of a New Phaco- matosis," Neurosurgery, Vol. 46, 2000, pp. 371-383. doi:10.1097/00006123-200002000-00021

[3] S. Robinson and A. R. Cohen, "Cowden Disease and Lhermitte-Duclos Disease: An Update. Case Report and Review of the Literature," Neurosurgical Focus, Vol. 20, No. 1, 2006, p. E6. doi:10.3171/foc.2006.20.1.7

[4] T. C. Tan and L.C Ho, "Lhermitte-Duclos Disease Associated with Cowden Syndrome," Journal of Clinical Neuroscience, Vol. 14, No. 8, 2007, pp. 801-805. doi:10.1016/j.jocn.2006.06.007

[5] B. Yağci-Küpeli, K. K. Oguz, M. A. Bilen, B. Yalçin and N. Akalan, "An Unusual Cause of Posterior Fossa Mass: Lhermitte-Duclos Disease," Journal of the Neurological Sciences, Vol. 290, No. 1-2, 2010, pp. 138-141. doi:10.1016/i.jns.2009.12.010

[6] C. Murray, P. Shipman and M. Khangure, "LhermitteDuclos Disease Associated with Cowden's Syndrome: Case Report and Literature Review," Australasian Radiology, Vol. 45, No. 3, 2001, pp. 343-346. doi:10.1046/j.1440-1673.2001.00933.x

[7] N. G. Rainov, H. J. Holzhausen and W. Burkert, "Dysplastic Gangliocytoma of the Cerebellum (Lhermitte-Duclos Disease)," Clinical Neurology and Neurosurgery, Vol. 97, No. 2, 1995, pp. 175-180. doi:10.1016/0303-8467(95)00017-E

[8] F. Barone, B. A. Noubari, A. Torrisi, S. Lanzafame and R. Tropea, "Lhermitte Duclos Disease and Cowden Disease: Clinical, Pathological and Neuroimaging Study of a Case," Journal of Neurosurgical Sciences, Vol. 44, No. 4, 2000, pp. 234-237.

[9] A. D. Nowak, A. H. Trost, A. Porr, A. Stolzle and C. B. Lumenta, "Lhermitte-Duclos Disease (Dysplastic Gangliocytoma of the Cerebellum)," Clinical Neurology and Neurosurgery, Vol. 103, No. 2, 2001, pp. 105-110. doi:10.1016/S0303-8467(01)00124-X

[10] S. Nagaraja, T. Powell, P. D. Griffiths and I. D. Wilkinson, "MR Imaging and Spectroscopy in Lhermitte-Duclos Disease," Neuroradiology, Vol. 46, No. 5, 2004, pp. 355358. doi:10.1007/s00234-004-1201-7

[11] E. Özkavukcu, P. S. Öztekin and E. I. Erden, "Preoperative Diagnosis of Lhermitte-Duclos Disease: MRI and 1H-MR Spectroscopy Findings," European Journal of Radiology Extra, Vol. 64, No. 3, 2007, pp. 83-86. doi:10.1016/j.ejrex.2007.08.005

[12] T. Faillot, J. Sichez, J. Brault, L. Capelle, M. Kujas, L. Bordi, et al., "Lhermitte-Duclos Disease (Dysplastic Gangliocytoma of the Cerebellum). Report of a Case and Review of the Literature," Acta Neurochirurgica, Vol. 105, No. 1-2, 1990, pp. 44-49. doi:10.1007/BF01664857

[13] C. C. Meltzer, J. G. Smirniotopoulos and R. V. Jones, "The Striated Cerebellum: An MR Imaging Sign in Lhermitte-Duclos DIsease (Dysplastic Gangliocytoma)," Radiology, Vol. 194, No. 3, 1995, pp. 699-703.

[14] L. J. Qian, J. R. Xu and L. Y. Zheng, "Neurological Picture. Lhermitte-Duclos Disease,"Journal of Neurology, Neurosurgery \& Psychiatry, Vol. 81, No. 3, 2010, pp. 255-256. doi:10.1136/jnnp.2009.182808 
[15] L. Zhou, L. Luo, X. Hui, C. You, Y. Yang and J. Xu "Three Adolescents with Lhermitte-Duclos Disease," Journal of Clinical Neuroscience, Vol. 16, No. 1, 2009, pp. 124-127. doi:10.1016/j.jocn.2008.02.023

[16] M. S. Yang, C. H. Kim, J. H. Cheong and J. M. Kim, "Lhermitte-Duclos Disease Presenting with Hydrocephalus," Acta Neurochirurgica Supplement, Vol. 113, 2012, pp. 161-165. doi:10.1007/978-3-7091-0923-6_32

[17] A. Afshar-Oromieh, H. Linhart, D. Podlesek, W. Schrempf, G. Schackert and D. Krex, "Postoperative Cerebellar Mutism in Adult Patients with Lhermitte-Duclos Disease," Neurosurgery Review, Vol. 33, No. 4, 2010, pp. 401-408. doi:10.1007/s10143-010-0278-1 